\title{
Composición del banco de semillas e invasión de Ligustrum lucidum en bosques costeros de la provincia de Buenos Aires, Argentina
}

\author{
Seed bank composition and Ligustrum lucidum \\ invasion in coastal forests in Buenos Aires, Argentina
}

\author{
María Virginia Eva Diaz Villa ${ }^{\text {a*, }}$, Nora Madanes ${ }^{\text {a,b }}$, Piedad M Cristiano ${ }^{\text {a,b }}$, Guillermo Goldstein ${ }^{\text {a }}$

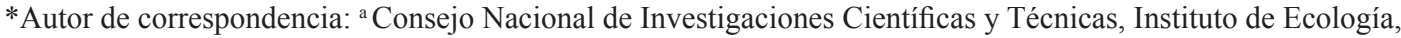 \\ Genética y Evolución de Buenos Aires, Laboratorio de Ecología Funcional, Ciudad Universitaria Pab. II, C.A.B.A, Argentina, \\ tel.: 5411 4576-3300,virginiadv@ege.fcen.uba.ar \\ ${ }^{\mathrm{b}}$ Universidad de Buenos Aires, Facultad de Ciencias Exactas y Naturales, Departamento de Ecología, \\ Genética y Evolución, Laboratorio de Ecología Funcional, C.A.B.A, Argentina.
}

\begin{abstract}
SUMMARY
Seed banks are key components of terrestrial ecosystems as they determine the future development of its vascular plants. In disturbed environments, seed banks can either favor regeneration of native species, or enhance colonization and expansion of exotic species. In Buenos Aires Province (Argentina), Celtis tala forests are the main wooded community. They have experienced strong anthropogenic disturbances that made them vulnerable to invasion from the exotic tree Ligustrum lucidum. The objective of this work was to study the seed bank composition in Celtis tala forests in "El Destino" Reserve (Magdalena, Buenos Aires) and its relation with the standing vegetation in areas with distinct degrees of invasion. Five plots were stablished in two wooded cordons, covering four types of environments. In each plot, a soil sample was taken and standing vegetation was studied. Seed bank composition was analyzed qualitatively and quantitatively during four months in a Ciudad Universitaria's greenhouse. Invaded environments were characterized by having less diversity, richness and evenness than those presented by non-invaded environments. Non-invaded environment's seed banks exhibited a larger proportion of herbaceous species while in invaded seed banks, Ligusrtrum lucidum dominated. This result shows that invasion has exceeded the threshold of reversibility, consolidating as neo-ecosystems dominated by L. lucidum.
\end{abstract}

Key words: invasiveness, diversity, glossy privet, Celtis tala.

\section{RESUMEN}

El banco de semillas es un componente clave de los ecosistemas terrestres ya que determina el desarrollo futuro de la estructura y composición de sus plantas vasculares. En ambientes disturbados, el banco de semillas puede favorecer la regeneración de las especies nativas o la colonización y expansión de especies exóticas. Los bosques dominados por Celtis tala constituyen la principal comunidad boscosa de la provincia de Buenos Aires (Argentina) y han experimentado fuertes perturbaciones antrópicas, convirtiéndose en susceptibles a la invasión por la especie exótica Ligustrum lucidum (ligustro). El objetivo del trabajo fue estudiar la composición del banco de semillas de los bosques de tala de la Reserva "El Destino" (Magdalena, Buenos Aires), su relación con la vegetación y distintas propiedades del suelo en áreas con distinto grado de invasión. Se establecieron cinco parcelas en dos cordones boscosos que abarcaron cuatro tipos de bosques (conservado, semi-conservado, ligustral e implantado) de donde se tomaron muestras de suelo y se relevó la vegetación. Se analizó cualitativa y cuantitativamente la composición del banco de semillas durante cuatro meses en el invernadero de Ciudad Universitaria de la Universidad de Buenos Aires. Los bosques invadidos se caracterizaron por presentar menor diversidad, riqueza y equitatividad que el resto de los bosques. Los bancos de semilla de los bosques conservado y semi-conservado presentaron mayor proporción de especies herbáceas mientras que en los bosques invadidos dominó L. lucidum. Estos resultados sugieren que el grado de invasión ha superado el umbral de irreversibilidad, consolidándose como neoecosistemas dominados por L. lucidum.

Palabras clave: banco de semillas invadido, relación banco de semilla-vegetación, Celtis tala.

\section{INTRODUCCIÓN}

El banco de semillas constituye un componente clave en la dinámica de las comunidades vegetales ya que representa un flujo continuo de aporte y pérdida de semillas que determina la densidad, la composición y la reserva genética del bosque (Simpson et al. 1989). Este banco puede constituir una fuente de propágulos capaz de regenerar la comunidad nativa luego de un disturbio y mantener la riqueza específica, o bien, constituir una fuente de potenciales especies invasoras. En bosques primarios, la composición del banco de semillas suele estar dominada 
por especies nativas pioneras mientras que en ambientes altamente antropizados, los bosques pueden estar constituidos casi enteramente por especies exóticas, reduciendo la posibilidad de las especies nativas de recuperarse (Kalesnik et al. 2012). En este sentido, el grado de asociación o la relación entre la composición del banco de semillas y la de la vegetación ha sido utilizado para predecir el potencial regenerativo de una comunidad vegetal luego de un disturbio (Albrecht y Pilgram 1997).

Los bosques dominados por Celtis tala Gill ex Planch, constituyen la principal comunidad boscosa nativa del NE de la provincia de Buenos Aires (Athor 2010). Estos bosques se distribuyen sobre suelos con buen drenaje y originalmente se extendían todo a lo largo de las barrancas del Paraná y de los bancos de conchilla producto de ingresiones y regresiones marinas y médanos muertos próximos al Río de la Plata y el Océano Atlántico, constituyendo prolongaciones de la provincia del espinal. También se encuentran sobre los médanos fósiles localizados en los bordes de lagunas de la Pampa Deprimida que fueron cubetas de deflación, relictos de un periodo árido del cuaternario (Cabrera 1976). Sin embargo, en la actualidad, estos bosques se encuentran en retroceso y están siendo reemplazados debido al impacto humano producido por la tala para extracción de leña y carbón, extracción de conchillas del suelo y actividades agrícolas, ganaderas y forestales (Athor 2010). Este impacto favoreció la introducción de especies exóticas, algunas de las cuales se comportan fuertemente como invasoras. Entre las principales especies leñosas invasoras se encuentran el ligustro (Ligustrum lucidum Ait.) y la ligustrina (Ligustrum sinense Lour.) (Athor 2010). Ligustrum lucidum es una de las especies exóticas de mayor distribución en la Argentina (Hoyos et al. 2010). Produce abundantes frutos que son comidos por las especies de aves nativas, por lo que contribuyen a la colonización de nuevos hábitats de esta especie. Es capaz de germinar tanto en condiciones de alta como baja intensidad de luz, a partir de frutos intactos o que fueron dispersados por aves (Aragón y Groom 2003, Rodríguez y Madanes 2010). Su capacidad de germinar en condiciones de baja luminosidad le permite establecerse en bosques poco disturbados. Una vez que alcanza el dosel puede desplazar a la vegetación nativa al crear condiciones de sombra que dificulta la regeneración de otras especies lo que resulta en la dominancia del ligustro por sobre las especies nativas (Hoyos et al. 2010). A su vez, se ha observado que la invasión por especies con dominancia estructural, como el ligustro, resulta en una disminución en la riqueza y densidad específicas, así como en un cambio en la composición específica de la comunidad. Los cambios inducidos por estas especies pueden incluso facilitar el establecimiento de nuevas especies exóticas, un efecto conocido como "fusión invasional" (Gioria et al. 2014, Ferreras et al. 2015). En consecuencia, las comunidades invadidas se han caracterizado por presentar mayor ri- queza y/o abundancia de semillas de especies exóticas (Gioria et al. 2012). También se ha observado una disminución en la acidez de los suelos y un significativo aumento del nitrógeno mineral disponible en sitios invadidos por la especie cogenérica Ligustrum sinense Lour. en el SE de EE.UU. (Lobe et al. 2014).

El objetivo de este trabajo es estudiar la composición del banco de semillas en áreas con distinto grado de conservación de los cordones boscosos del Parque Costero del Sur (PCS) y su relación con las propiedades del suelo y la vegetación. La hipótesis de trabajo es que la dominancia estructural de L. lucidum está asociada a diferencias en los bancos de semillas y en las propiedades del suelo, en comparación con los bosques conservados. En particular, se espera observar diferencias en la composición específica, menor riqueza y mayor densidad de semillas de especies exóticas en los bancos de semilla de bosques con algún grado de invasión.

\section{MÉTODOS}

Área de estudio. El trabajo se llevó a cabo en la Reserva de Flora y Fauna autóctonas "El Destino" ubicada en el Partido de Magdalena, Provincia de Buenos Aires, Argentina $\left(35^{\circ} 08^{\prime} \mathrm{S}, 57^{\circ} 25^{\prime} \mathrm{O}\right)$. La misma comprende 2.400 ha y forma parte de la Reserva de la Biósfera "Parque Costero del Sur" (MAB-UNESCO) de 26.581 ha de superficie (Athor 2010). Estos talares se ubican en los cordones de depósitos calcáreos paralelos a la costa, que se originaron durante las ingresiones marinas cuaternarias. Son suelos Rendoles constituidos por materiales gruesos que presentan un buen drenaje y aireación, favoreciendo la actividad biológica y la humificación (Athor 2010). Estos cordones alternan con áreas deprimidas de materiales finos en superficie. El clima es templado y presenta bajas amplitudes térmicas diarias y estacionales debido a la influencia del Río de La Plata y la cercanía del océano Atlántico (Athor 2010). La temperatura media anual se encuentra entre $15^{\circ} \mathrm{C}$ y $17^{\circ} \mathrm{C}$. Los valores anuales de precipitación varían entre 850 y $1.065 \mathrm{~mm}$ con máximos de ocurrencia durante el otoño y la primavera (Athor 2010). La zona de estudio se encuentra en el borde de la Pampa Deprimida, con escasa pendiente y atravesada por cursos de agua que desembocan en el Río de la Plata, la Bahía de Samborombón y en el Océano Atlántico.

Los talares pueden estar dominados por Celtis tala, Scutia buxifolia Reissek o presentar codominancia de ambas especies (Athor 2010). El sotobosque fue descripto por Cagnoni et al. (1996). En el mismo están presentes Sida rhombifolia L. y Pavonia malvácea (Vell.) Krap. et Crist. Sobre árboles y arbustos crecen enredaderas tales como Clematis denticulata Vell., Passiflora caerulea L., Parthenocissus quinquefolia (L.) Planch., Tropaeolum pentafilum Lam., entre otras. El estrato herbáceo está constituido por especies propias del talar como son Oplismenus setarius (Lam.) Roem. et Schult., Parietaria debilis G. Forst, Euphorbia portulacoides L., y de otras comunidades vecinas. 
La composición del bosque presenta una variación espacial muy definida donde $C$. tala domina en los cordones más alejados del río y $S$. buxifolia en aquellos más cercanos (Ribichich 1996). Esta distribución podría reflejar diferencias en los requerimientos ambientales de dichas especies.

Análisis de la vegetación. Se realizó un muestreo de campo en septiembre del 2014 (primavera). Se ubicaron cinco parcelas de $10 \mathrm{~m} \times 10 \mathrm{~m}$ a lo largo de dos cordones boscosos de la reserva, abarcando cuatro tipos de bosques: conservado ( 2 parcelas), semi-conservado (3 parcelas), ligustral (2 parcelas) e implantado (3 parcelas) (figura 1). El número de parcelas por ambiente fue diferente debido a la longitud de los cordones y a la distribución de los ambientes en los mismos. Los bosques conservados y semiconservados, se ubican sobre un mismo cordón cercano al río. El bosque conservado corresponde a un sector donde se realizan acciones permanentes de conservación del ambiente de talar. El bosque semi-conservado, por su parte presenta una composición específica similar al conservado aunque en el estrato arbustivo se observa una gran cantidad de juveniles de ligustro, indicando un estado de invasión intermedio. En un segundo cordón, más alejado del río, se ubican los bosques ligustral e implantado. El ligustral corresponde a un sector del bosque completamente dominado en todos sus estratos por L. lucidum. Por último, el bosque implantado se ubica en los alrededores de los jardines de la reserva, y consiste en un bosque implantado en la década de 1920, con ejemplares de nogales, cedros y robles.

En cada parcela, en los estratos arbóreo y arbustivo, se identificaron las especies y se contaron los individuos por especie. Para el relevamiento del estrato herbáceo se estable- ció una sub-parcela de $1 \mathrm{~m} \times 1 \mathrm{~m}$ ubicada en una esquina de la parcela. En cada sub-parcela se estimó la abundancia-cobertura de las especies vasculares según la escala de BraunBlanquet modificada (Mueller-Dombois y Ellenberg 1974) y el porcentaje de broza y de suelo desnudo. Se confeccionó un herbario para la posterior identificación de las especies que no pudieron ser determinadas en campo. Las especies identificadas en campo y herborizadas fueron corroboradas utilizando floras regionales (Cabrera 1993) y consultas a especialistas. Las especies fueron clasificadas por familia siguiendo a Cabrera (1993) y luego se agruparon en función del tipo y ciclo biológico, su fenología y origen (Zuloaga et al. 2015).

En cada ambiente, se calculó la diversidad a través del índice de Shannon-Wiener, la equitatividad y la riqueza (Magurran 1988). También se calculó el índice de invasión (número de especies exóticas/número de especies totales) y la cobertura media de especies exóticas (sumatoria de la cobertura media de cada especie exótica/sumatoria de cobertura media del total de especies) en cada estrato.

Análisis del banco de semillas y suelo. En los vértices y en el centro de cada parcela se tomaron cinco muestras de suelo en octubre de 2014, antes de producirse la fructificación de las principales especies leñosas nativas. Por lo tanto, las semillas de estas especies presentes en el suelo corresponden al período de fructificación anterior. Las muestras no se sometieron a estratificación térmica ya que pasaron naturalmente por este proceso en el área de estudio durante el invierno. Cada una de las muestras colectadas fue colocada en una bandeja plástica de $15 \times 20 \mathrm{~cm}$ en el vivero de la Facultad de Ciencias Exactas y Naturales, Ciudad Universitaria, Ciudad Autónoma de Buenos Aires

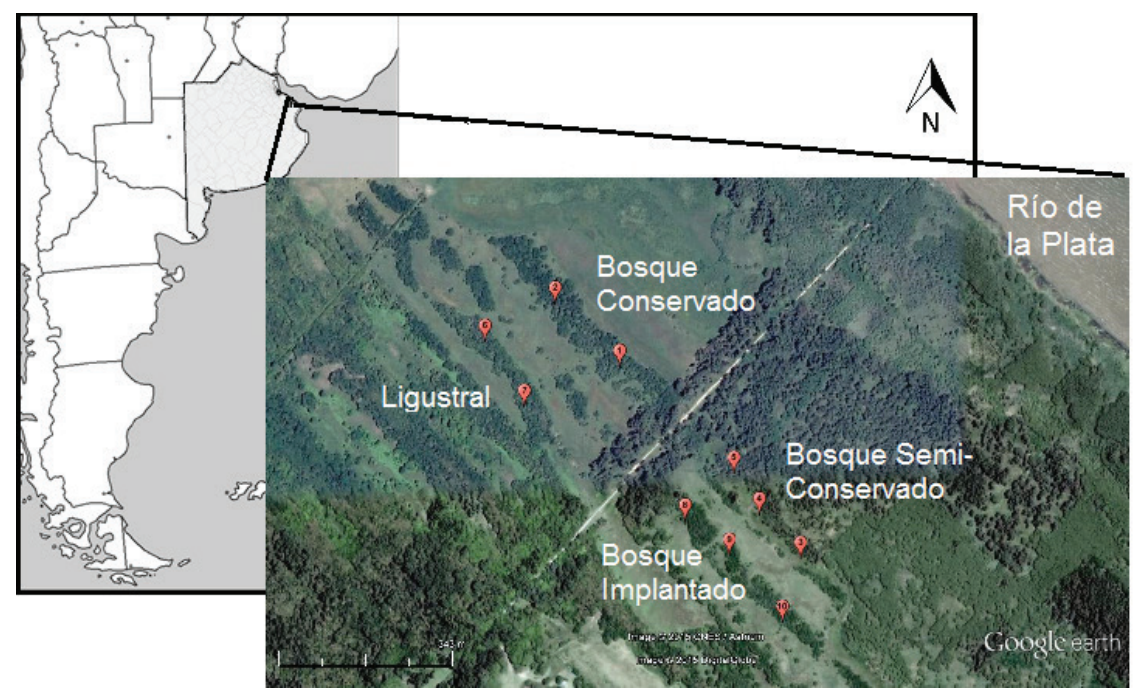

Figura 1. Imagen satelital de la Reserva de Flora y Fauna autóctonas "El Destino". Se muestran los 10 sitios de muestreo en dos cordones boscosos de la misma. Fecha de la imagen: diciembre de 2015. Fuente: Google Earth. Las flechas punteadas indican la extensión de los cordones.

Satellite image of "El Destino" Reserve. The ten plots over the two wooded cordons are shown. Date of image: December 2015. Source: Google Earth. Dotted lines show each wooded cordon extension. 
constituyendo un total de 50 bandejas. Éstas fueron regadas diariamente para mantener la humedad y estuvieron cubiertas con una media sombra de manera de simular las condiciones lumínicas del sotobosque.

En cada bandeja se registró cada dos días la composición y abundancia de las plántulas desde el estado de cotiledón y se tomó registro fotográfico de las mismas. La identificación taxonómica de las plántulas implicó que éstas alcanzaran un estado vegetativo en que fuesen factibles de identificar ya sea por comparación con la descripción en bibliografía específica (Cabrera 1993) o mediante consultas a especialistas. La clasificación se realizó hasta el máximo nivel jerárquico taxonómico posible. En cada parcela se registró la densidad total de semillas germinadas por metro cuadrado y la densidad de semillas germinadas por especie y se determinó la composición del banco según sus formas de vida, ciclo biológico y origen. Se siguió el desarrollo de las plántulas de cada bandeja durante 16 semanas. Para evitar competencia por interferencia, las plántulas germinadas se removieron cada semana de las bandejas de germinación, y se siguió su desarrollo en nuevas bandejas o maceta individuales. Se realizaron curvas de germinación acumulada en el total de bandejas de cada parcela, y se estableció el tiempo final una vez que las curvas alcanzaron una meseta.

Para el análisis de las variables edáficas se extrajo una muestra al azar dentro de cada parcela con un barreno circular de $10 \mathrm{~cm}$ de diámetro y $5 \mathrm{~cm}$ de profundidad de PVC (volumen total 78,54 $\mathrm{cm}^{3}$ ). Las muestras fueron secadas en estufa a $60{ }^{\circ} \mathrm{C}$ hasta obtener un peso constante. Se estimó el $\mathrm{pH}$ utilizando papel $\mathrm{pH}$ sobre una muestra de tierra humedecida de cada parcela. El porcentaje de humedad (H \%) y de materia orgánica del suelo (MOS \%) se estimaron a partir del método de pérdida por ignición (Davies 1974).

Comparación del banco de semilla con variables edáficas y la vegetación. Se realizó un análisis de correspondencia canónico (CCA) para analizar la relación entre la vegetación y las variables edáficas empleando el programa CANOCO 4.5. Se consideraron los valores de densidad del estrato arbóreo y arbustivo y, para la vegetación herbácea, el valor promedio de los intervalos de abundanciacobertura de la escala de Braun-Blanquet de cada muestra. Previamente, se realizó una transformación logarítmica de los datos (Escudero et al. 1994). Se empleó el índice de Sorensen (QS) para calcular la similitud general entre las especies presentes en los bancos de semillas y la vegetación para cada uno de los bosques definidos (Magurran 1988). Se realizó un ANDEVA de un factor para comparar las variables $\mathrm{pH}, \mathrm{MOS} \%, \mathrm{H} \%$, CMex Árboles, CMex Arbustos e índice de invasión, previa comprobación de los supuestos (homogeneidad de varianzas y normalidad), con $\alpha=0,05$. Las comparaciones múltiples se realizaron mediante la prueba de Tukey. Para las variables CMex Herbáceas y QS, que no cumplieron el supuesto de homogeneidad de varianzas, se realizó una prueba de Kruskal-Wallis. Las pruebas se realizaron utilizando el programa InfoStat.

\section{RESULTADOS}

Los bosques conservado y semi-conservado, ubicados sobre el cordón más cercano al río, se encontraron dominados por especies arbóreas nativas como Scutia buxifolia $(27,5$ y $40 \%$, respectivamente) y Celtis tala (10 y $40 \%$, respectivamente). A su vez, se registraron especies acompañantes típicas del talar, como Jodina rhombifolia (Hook. et Arn.) Reissek, Schinus longifolius (lindl.) Speg. y Phytolacca dioica L. en ambos bosques. En el sotobosque se registraron especies típicas del ambiente del talar tanto nativas (Oplismenus setarius, Sida rhombifolia, Passiflora caerulea y Parthenocissus quinquefolia), así como exóticas (Stellaria media (L.) Cirillo, Cirsium vulgare (Savi.) Ten. y Dipsacus fullanum L.), con una cobertura de herbáceas del 86 y $80 \%$, respectivamente. Además, se registró la presencia en el estrato arbustivo de Lonicera japónica Thunb. y Rubus ulmifolius Schott. Los bosques ligustral e implantado, en cambio, se encontraron dominados por la especie exótica L. lucidum ( 90 y $80 \%$, respectivamente), acompañada por un muy bajo porcentaje de especies nativas. A su vez, estos bosques presentaron una alta cobertura del dosel (95 y 96,7 \%, respectivamente) y una menor cobertura de especies herbáceas en el sotobosque (73 y 59,75\%, respectivamente) (cuadro 1).

El análisis canónico de correspondencia evidenció que las variables edáficas (cuadro 2) permiten explicar el $87 \%$ de la variabilidad en la composición florística de los bosques ( $43 \%$ la materia orgánica, $25 \%$ el pH y $19 \%$ el porcentaje de humedad) (figura 2). El primer eje explicó el $59,8 \%$ de la varianza total. Hacia los valores positivos de este eje se ubicaron las parcelas que presentaron los mayores porcentajes de materia orgánica y menor $\mathrm{pH}$, mientras que hacia los valores negativos la tendencia se invirtió. El segundo eje explicó el 22,7 \% de la varianza y se relacionó con el contenido de agua en el suelo, ubicando a las parcelas con mayor porcentaje de humedad hacia el semieje negativo. La distribución espacial de las parcelas, determinada por estas variables, permitió distinguir dos grupos. El grupo A (bosque conservado), con alta riqueza de especies herbáceas, se asoció a un mayor contenido de materia orgánica y acidez y se distribuyó hacia el extremo positivo del primer eje. El grupo B (bosques con distinto grado de invasión) se distribuyó hacia el extremo negativo del primer eje y se caracterizó por presentar un menor contenido de materia orgánica en el suelo y $\mathrm{pH}$ neutro. Las especies arbóreas y arbustivas exóticas $L$. lucidum, $L$. sinense, Acer negundo L., Juglans regia L., Platanus hispánica Mill. y Populus nigra L. aparecieron asociadas a este grupo. El contenido de humedad del suelo no resultó determinante en la composición del estrato herbáceo de los sitios dado que las parcelas correspondientes al bosque conservado presentaron diferencias en el contenido de humedad del suelo pero no en la composición y riqueza específica (figura 2). 
Cuadro 1. Especies relevadas en los cordones boscosos de la Reserva "El Destino". Estrato en el que se observó: arbóreo (A), arbustivo (Ar) y herbáceo (H); tipo biológico: árbol (A), arbusto (Ar), herbácea latifoliada (HL), herbácea graminiforme (HG), herbácea equisetoide (HE) o enredadera (E); ciclo biológico: anual (A) o perenne (P); origen: exótica $(\mathrm{E})$ o nativa $(\mathrm{N})$; bosque: conservado $(\mathrm{C})$, semi-conservado (SC), ligustral (L) e implantado (I).

Species found in wooded cordons in "El Destino" Reserve. Species surveyed in wooded cords in "El Destino" Reserve. Stratum in which they were observed: arboreal (A), shrub (Ar) and herbaceous (H); Biological type: tree (A), bush (Ar), broadleaved herbaceous (HL), gramineous herbaceous (HG), equisetoid herbaceous (HE) or creeper (E); Biological cycle: annual (A) or perennial (P); Origin: exotic (E) or native $(\mathrm{N})$; forest type: canned (C), semi-conserved (SC), ligustral (L) and implanted (I).

\begin{tabular}{|c|c|c|c|c|c|c|c|}
\hline Familia & Especie & $\begin{array}{c}\text { Acrónimo } \\
\text { CCA }\end{array}$ & Estrato & $\begin{array}{c}\text { Tipo } \\
\text { biológico }\end{array}$ & $\begin{array}{c}\text { Ciclo } \\
\text { de vida }\end{array}$ & Origen & Bosque \\
\hline Aceraceae & Acer negundo L. & Acene & $\mathrm{H}$ & A & $\mathrm{P}$ & $\mathrm{E}$ & L-I \\
\hline Anacardiaceae & Schinus longifolius (Lindl.) Speg. & Schlo & A & A & $\mathrm{P}$ & $\mathrm{N}$ & C- SC- L \\
\hline \multirow[t]{3}{*}{ Apiaceae } & Apium leptophyllum (Pers.) F. Muell. & Apile & $\mathrm{H}$ & HL & A & $\mathrm{N}$ & $\mathrm{C}$ \\
\hline & Hydrocotyle vulgaris $\mathrm{L}$. & $H y d v u$ & $\mathrm{H}$ & HL & $\mathrm{P}$ & $\mathrm{N}$ & $\mathrm{C}$ \\
\hline & Torilis nodosa (L.) Gaertn. & Torno & $\mathrm{H}$ & HL & A & $\mathrm{E}$ & $\mathrm{C}$ \\
\hline \multirow[t]{2}{*}{ Asteraceae } & Baccharis articulata (Lam.) Pers. & Bacar & Ar-H & $\mathrm{Ar}$ & $\mathrm{P}$ & $\mathrm{N}$ & $\mathrm{C}$ \\
\hline & Cirsium vulgare (Savi.) Ten. & Cirvu & $\mathrm{H}$ & $\mathrm{HL}$ & $\mathrm{A}$ & $\mathrm{E}$ & $\mathrm{C}$ \\
\hline \multirow[t]{6}{*}{ Asteraceae } & Senecio madagascariensis Poir. & Senma & $\mathrm{H}$ & HL & $\mathrm{P}$ & $\mathrm{E}$ & $\mathrm{C}$ \\
\hline & Solidago chilensis Meyen. & Solch & $\mathrm{H}$ & $\mathrm{Ar}$ & $\mathrm{P}$ & $\mathrm{N}$ & $\mathrm{C}$ \\
\hline & Sonchus asper (L.) Hill & Sonas & $\mathrm{H}$ & $\mathrm{HE}$ & $\mathrm{P}$ & $\mathrm{N}$ & $\mathrm{C}$ \\
\hline & Taraxacum officinale G. Weber ex F. H. Wigg. & Tarof & $\mathrm{H}$ & HL & $\mathrm{P}$ & $\mathrm{E}$ & $\mathrm{C}$ \\
\hline & Verbecina encelioides (Cav.) Benth. Y Hook F. ex A. Gray. & Veren & $\mathrm{H}$ & HL & A & $\mathrm{N}$ & $\mathrm{SC}$ \\
\hline & Xanthium cavanillesii Schouw. & Xanca & $\mathrm{H}$ & HL & A & $\mathrm{E}$ & $\mathrm{C}-\mathrm{SC}$ \\
\hline Caprifoliaceae & Lonicera japónica Thunb. & Lonca & Ar-H & $\mathrm{E}$ & $\mathrm{P}$ & $\mathrm{E}$ & $\mathrm{C}$ \\
\hline Caryophyllaceae & Stellaria media (L.) Cirillo & Steme & $\mathrm{H}$ & HL & A & $\mathrm{E}$ & $\mathrm{SC}$ \\
\hline Commelinaceae & Tradescantia fluminensis Vell. & Traft & $\mathrm{H}$ & $\mathrm{HL}$ & $\mathrm{P}$ & $\mathrm{N}$ & L-I \\
\hline Cyperaceae & Carex divulsa Stokes & Cardi & $\mathrm{H}$ & $\mathrm{HE}$ & $\mathrm{P}$ & $\mathrm{E}$ & $\mathrm{SC}$ \\
\hline Dipsacaceae & Dipsacus fullonum $\mathrm{L}$. & Dipfu & $\mathrm{H}$ & $\mathrm{HL}$ & A & $\mathrm{E}$ & $\mathrm{C}$ \\
\hline Juglandaceae & Juglans regia $\mathrm{L}$. & Jugre & A-Ar-H & A & $\mathrm{P}$ & $\mathrm{E}$ & I \\
\hline Malvaceae & Abutilon grandifolium (Willd.) Sweet & Malv & $\mathrm{H}$ & $\mathrm{HL}$ & $\mathrm{P}$ & $\mathrm{N}$ & $\mathrm{C}$ \\
\hline Meliaceae & Melia azedarch $\mathrm{L}$. & Melaz & $\mathrm{Ar}$ & A & $\mathrm{P}$ & $\mathrm{E}$ & $\mathrm{C}$ \\
\hline Oleaceae & Ligustrum lucidum Ait. & Liglu & A-Ar-H & A & $\mathrm{P}$ & $\mathrm{E}$ & SC-L-I \\
\hline Orobanchaceae & Gerardia communis Cham. Y Schltdl. & Gerco & $\mathrm{H}$ & $\mathrm{Ar}$ & $\mathrm{P}$ & $\mathrm{N}$ & $\mathrm{C}$ \\
\hline Oxalidaceae & Oxalis violácea Savigny & Oxavi & $\mathrm{H}$ & $\mathrm{HL}$ & $\mathrm{P}$ & $\mathrm{N}$ & C-SC \\
\hline Passifloraceae & Passiflora caerulea $\mathrm{L}$. & Pasca & $\mathrm{H}$ & $\mathrm{E}$ & $\mathrm{P}$ & $\mathrm{N}$ & $\mathrm{SC}$ \\
\hline Phytolaccaceae & Phytolacca dioica $\mathrm{L}$. & Phydi & A & A & $\mathrm{P}$ & $\mathrm{N}$ & $\mathrm{L}$ \\
\hline Plantaginaceae & Plantago lanceolata $\mathrm{L}$. & Plala & $\mathrm{H}$ & HL & $\mathrm{P}$ & $\mathrm{E}$ & C-SC \\
\hline Platanaceae & Platanus hispánica Mill. Ex Muenchh & Plahi & A & A & $\mathrm{P}$ & $\mathrm{E}$ & I \\
\hline \multirow[t]{4}{*}{ Poaceae } & Oplismenus setarius (Lam.) Roem. Y Schult. & Oplse & $\mathrm{H}$ & $\mathrm{HG}$ & $\mathrm{P}$ & $\mathrm{N}$ & $\mathrm{SC}$ \\
\hline & Paspalum humanii Parodi & Pasp & $\mathrm{H}$ & HG & $\mathrm{P}$ & $\mathrm{N}$ & $\mathrm{C}$ \\
\hline & Роа апиа $\mathrm{L}$. & Poa & $\mathrm{H}$ & $\mathrm{HG}$ & A & $\mathrm{E}$ & C-SC \\
\hline & Nassella hyalina (Nees.) Barkworth & Stihy & $\mathrm{H}$ & $\mathrm{HG}$ & $\mathrm{P}$ & $\mathrm{N}$ & $\mathrm{C}$ \\
\hline Rhamnaceae & Scutia buxifolia Reissek & Scubu & $\mathrm{A}-\mathrm{Ar}$ & A & $\mathrm{P}$ & $\mathrm{N}$ & C-SC \\
\hline Rosaceae & Rubus ulmifolius Schott. & Rubul & $\mathrm{H}$ & Ar & $\mathrm{P}$ & $\mathrm{E}$ & $\mathrm{L}$ \\
\hline Salicaceae & Populus alba $\mathrm{L}$. & Pop & A & A & $\mathrm{P}$ & $\mathrm{E}$ & L-I \\
\hline Santalaceae & Jodina rhombifolia (Hook. Y Arn.) Reissek & Jodrh & A-Ar-H & A & $\mathrm{P}$ & $\mathrm{N}$ & SC-L \\
\hline \multirow[t]{2}{*}{ Solanaceae } & Salpichroa origanifolia (Lam.) Baill. & Salor & $\mathrm{H}$ & HL & $\mathrm{P}$ & $\mathrm{N}$ & $\mathrm{SC}$ \\
\hline & Solanum pseudocapsicum L. & Solps & $\mathrm{H}$ & HL & $\mathrm{P}$ & $\mathrm{E}$ & $\mathrm{C}$ \\
\hline Ulmaceae & Celtis tala Gill. ex Planch & Celta & A & A & $\mathrm{P}$ & $\mathrm{N}$ & C-SC-I \\
\hline Vitaceae & Parthenocissus quinquefolia (L.) Planch & Parqu & $\mathrm{H}$ & E & $\mathrm{P}$ & $\mathrm{E}$ & C-C-L-I \\
\hline
\end{tabular}


Cuadro 2. Variables edáficas, índice de invasión y cobertura media de especies exóticas (CMex) en cada estrato de los bosques de la Reserva "El Destino". F y H corresponden a los estadísticos de las pruebas de ANDEVA de un factor y de Kruskal-Wallis realizadas en cada caso. Se informa el $P$-valor obtenido en cada comparación.

Edaphic variables, Invasive index and average cover of exotic species in each environment of the wooded cordon in "El Destino" Reserve. $\mathrm{F}$ and $\mathrm{H}$ correspond to statistical tests of one way ANOVA and Krukal-Wallis made in each case. The $P$-value obtained in each comparison is informed.

\begin{tabular}{lcccrrr}
\hline \multicolumn{1}{c}{ Bosque } & Conservado & Semi- conservado & Ligustral & Implantado & F/H & $P$-valor \\
\hline $\mathrm{pH}$ & $5,5 \pm 0,05$ & $6 \pm 0,29$ & $6,5 \pm 0,5$ & $6,5 \pm 0,29$ & 1,86 & 0,2373 \\
\% Humedad & $28,84 \pm 5,54$ & $28,54 \pm 3,46$ & $29,72 \pm 4,03$ & $28,98 \pm 0,94$ & 0,02 & 0,9957 \\
$\%$ MOS & $25,03 \pm 0,51$ & $18,92 \pm 1,47$ & $16,24 \pm 2,48$ & $18,97 \pm 1,54$ & 4,21 & 0,0636 \\
Índice de invasión (\%) & $44,14 \pm 0,03$ & $36,94 \pm 0,02$ & $83,33 \pm 0,17$ & $75,00 \pm 0,14$ & 4,17 & 0,0648 \\
CMex arbóreo (\%) & $0,00 \mathrm{a}$ & $0,00 \mathrm{a}$ & $95,20 \pm 0,05 \mathrm{~b}$ & $90,70 \pm 0,06 \mathrm{~b}$ & 171,41 & $<0,0001$ \\
CMex arbustivo (\%) & $28,50 \pm 0,01 \mathrm{a}$ & $74,10 \pm 0,12 \mathrm{ab}$ & $100,0 \mathrm{~b}$ & $90,00 \pm 0,1 \mathrm{~b}$ & 8,11 & 0,0156 \\
CMex herbáceo (\%) & $11,00 \pm 0,02$ & $33,00 \pm 0,27$ & $85,80 \pm 0,14$ & $97,70 \pm 0,02$ & 5,90 & 0,0869 \\
Índice de similitud (\%) & $25 \mathrm{ab}$ & $31,75 \pm 3,17 \mathrm{~b}$ & $22,5 \pm 2,5 \mathrm{a}$ & $42,22 \pm 12,37 \mathrm{ab}$ & 6,67 & 0,0356 \\
\hline
\end{tabular}

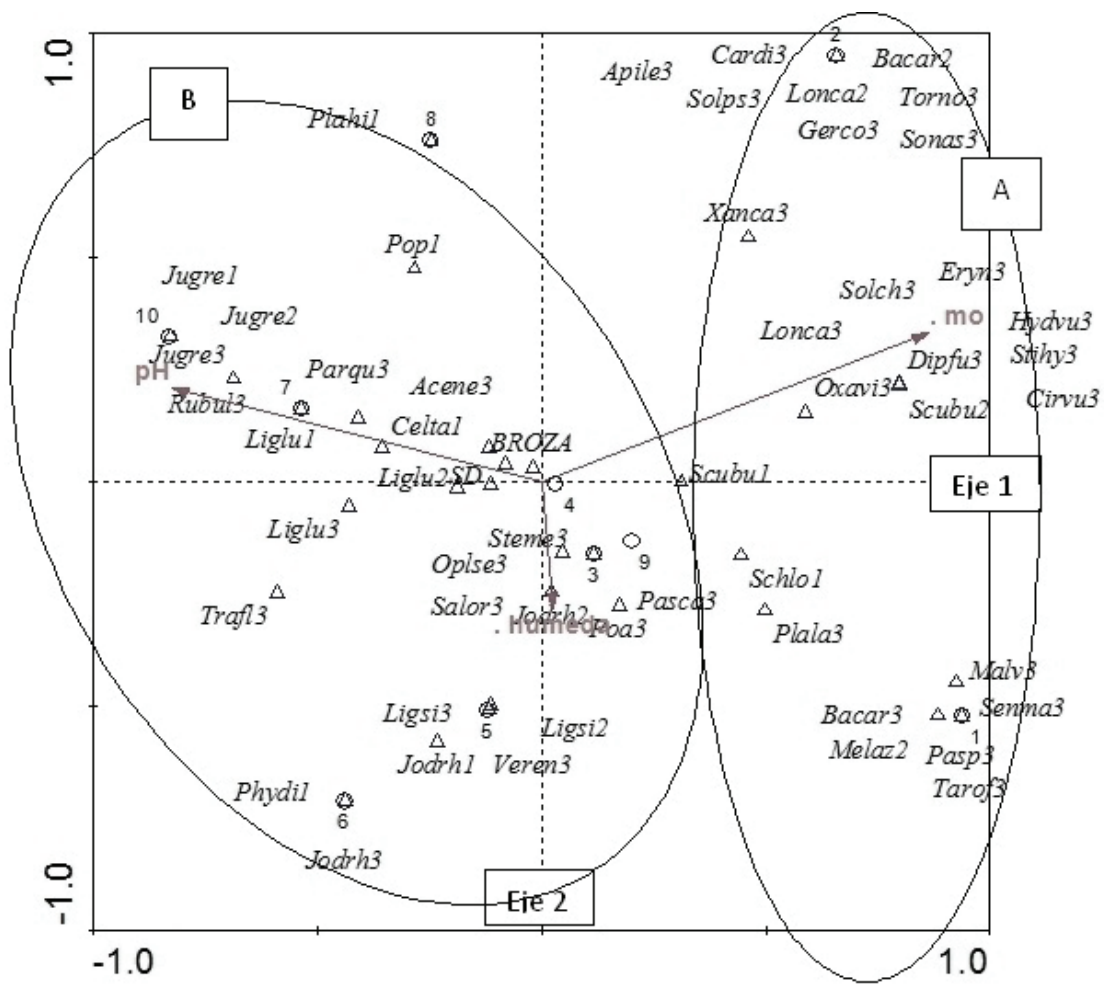

Figura 2. Análisis canónico de correspondencia (CCA) en el cual se incluyeron las variables edáficas como variables explicativas de la variabilidad en la composición florística de las parcelas relevadas en los cordones boscosos de la Reserva "El Destino". Además se señalan con un círculo los dos grupos propuestos, A y B. El grupo A se compone por las parcelas del bosque conservado mientras que el grupo B se constituye por los sitios que presentaron algún grado de invasión por L. lucidum. Los valores de cobertura media se transformaron a logaritmo neperiano. Para los ejes 1 y 2 : autovalores, 0,518 y 0,197; varianza explicada, 59,8 y 22,7 \%, respectivamente. Referencias: mo, contenido de materia orgánica del suelo, húmeda: contenido de agua en suelo, SD, suelo desnudo. Especies: ver cuadro 2. Los números que acompañan hacen referencia al estrato donde se encontró: 1, estrato arbóreo; 2, estrato arbustivo; 3 , estrato herbáceo. $\Delta$ Identifica especies, $\bigcirc$ parcelas $\mathrm{y} \rightarrow$ variables edáficas.

Canonic Correspondence Analysis where edaphic variables were included as explicative variables in the floristic composition of sampled plots. The two proposed groups, A and B, are shown with circles. Group A includes plots from conserved environment while Group B includes every plot that exhibited some degree of invasion by L. lucidum. Average cover was transformed into Napierian logarithm. For axes 1 and 2: eigenvalues, 0.518 and 0.197 ; explained variance, 59.8 and $22.7 \%$ respectively. References: edaphic variables measured in each plot: mo: organic matter content, humeda: water content and pH. Species: see table 2. Companion numbers make reference to stratum: 1, canopy layer; 2 , shrub layer; 3 , herbaceous layer. $\Delta$ Identify species, $\bigcirc$ plots and $\rightarrow$ edaphic variables. 
En cuanto a la riqueza, equitatividad y diversidad, se registró una disminución de estos atributos comunitarios en los bosques con mayor grado de invasión (figura 3 ). La riqueza de especies presentó una marcada tendencia decreciente entre el bosque conservado y el bosque implantado, mientras que la diversidad y la equitatividad presentaron valores máximos en los bosques conservado y semi-conservado y mínimos en el ligustral y el bosque implantado (figura 3).

Los bosques conservado y semi-conservado presentaron índices de invasión menores al $50 \%$. La cobertura media de especies exóticas (CMex) fue mayor en los tres estratos (arbóreo, arbustivo y herbáceo) del bosque semiconservado que los del bosque conservado. En los bosques con mayor grado de invasión (ligustral e implantado), se registraron índices de invasión mayores al $75 \%$. En estos dos bosques, la CMex es cercana al $100 \%$ en todos los estratos, y los mayores valores fueron registrados en los estratos arbóreo y arbustivo del ligustral (cuadro 2).

En el banco de semillas se registraron un total 29 especies, las cuales pertenecieron a 13 familias, siendo las familias más numerosas: Asteraceae con cinco especies (22,73\% del total) y Apiaceae y Malvaceae con tres especies cada una $(13,64 \%)$. Siete especies no pudieron ser determinadas ya que solo se desarrollaron hasta el estado de cotiledón y cuatro especies solo se determinaron hasta el nivel de familia. Del total de especies determinadas $(65,5 \%)$, el 10,34 \% correspondió a especies arbóreas, el 79,31\% a herbáceas latifoliadas, el 3,45\% a herbáceas graminiformes y el 6,89 \% herbáceas equisetoideas. El 57,9\% del total de especies identificadas fueron especies nativas

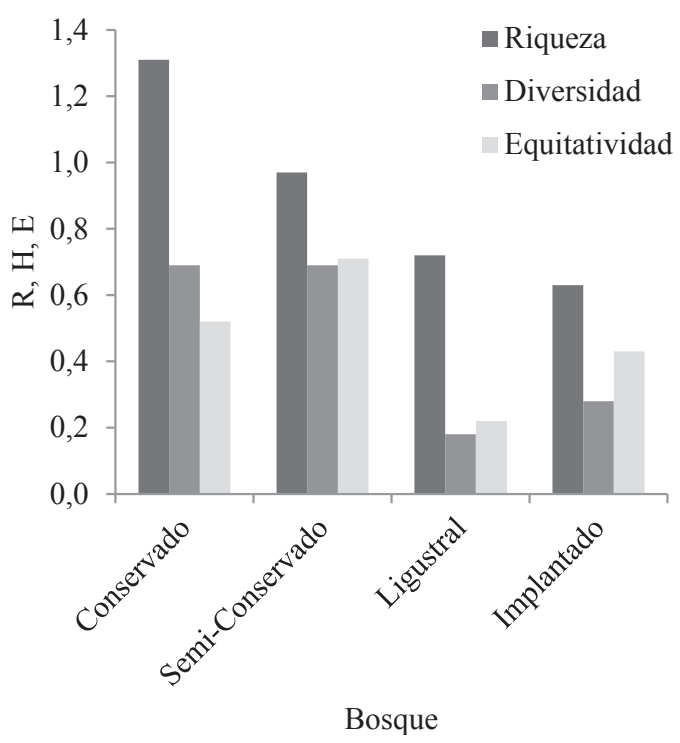

Figura 3. Diversidad (H'), riqueza (R) y equitatividad (E) para los distintos bosques de los cordones boscosos de la Reserva "El Destino".

Diversity (H'), Richness (R) and Evenness (E) for each environment in forests from "El Destino" Reserve. y el $42,1 \%$ exóticas. La mayor parte de las especies nativas identificadas correspondieron a especies herbáceas latifoliadas y graminiformes, mientras que entre las especies arbóreas dominaron las especies exóticas (figura 4). El índice de similitud de Sorensen (QS) para el total de especies entre los bancos de semilla y la vegetación fue de $40 \%$. El ligustral presentó la menor similitud, mientras que la mayor similitud se observó en el bosque implantado (cuadro 2).

Se obtuvo un total de 7.826 semillas germinadas $\mathrm{dm}^{-3}$, de las cuales 2.809 correspondieron a L. lucidum. Mientras que en los bosques conservado y semi-conservado se registró una mayor germinación de especies herbáceas, la relación se invierte en los bosques ligustral e implantado, donde germinaron mayor cantidad de semillas arbóreas, en particular de Ligustro (figura 5).

\section{DISCUSIÓN}

El análisis de la estructura y los cambios que se producen en los bancos de semilla durante las invasiones constituye un punto crítico para predecir la persistencia de una especie exótica en un ambiente determinado, así como para comprender las consecuencias a largo plazo en las comunidades nativas y en el funcionamiento del ecosistema (Gioria et al. 2012). En este estudio, L. lucidum, presentó la mayor densidad de semillas germinadas en los bosques invadidos (implantado y ligustral), seguida por la especie nativa Oplismenus setarius. Además, se observó una pre-

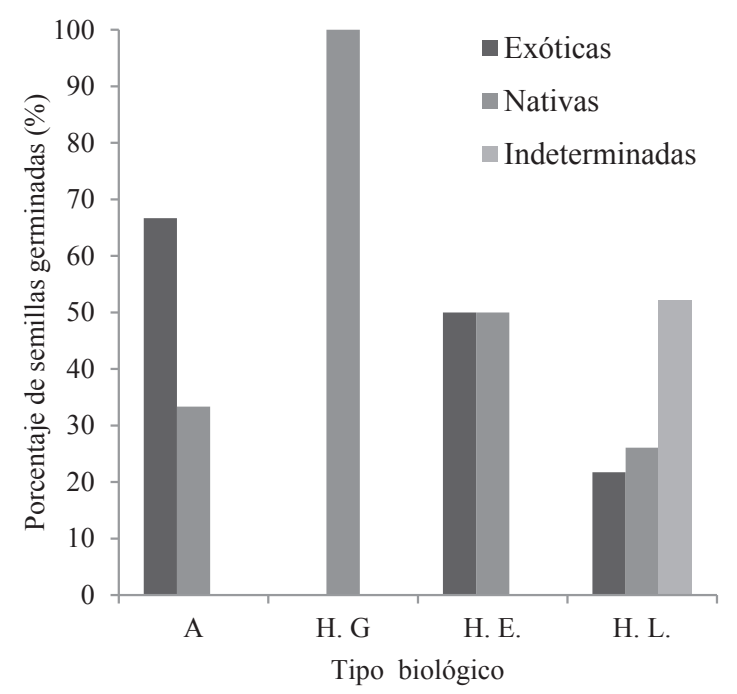

Figura 4. Proporción de especies nativas, exóticas e indeterminadas relevadas en los bancos de semilla de la Reserva "El Destino" en función de su tipo biológico, donde A corresponde a Árboles, H.G corresponde a Herbáceas graminiformes, H. E, Herbáceas equisetoideas y H. L, Herbáceas latifoliadas.

Native, exotic and undetermined species proportion found in "El Destino" Reserve's seed banks, according to its biological type, where A corresponds to trees, HG is for gramineous herbaceous, HE to equisetoide herbaceous and HL to broadleaf herbaceous. 


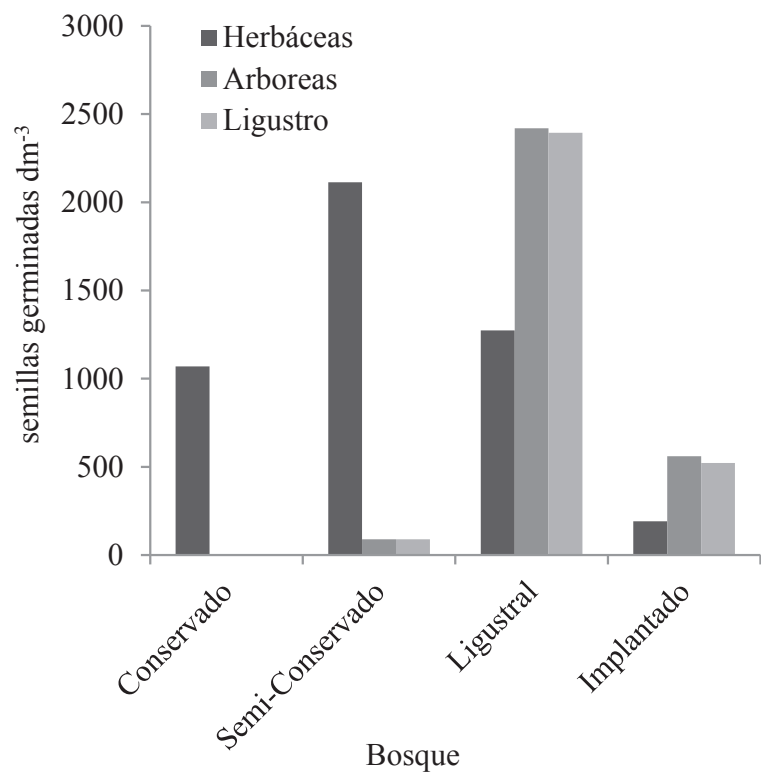

Figura 5. Número de semillas germinadas por tipo biológico y de ligustro en cada tipo de bosque.

Number of seeds germinated by biological type and privet seeds in each type of forest.

valencia de especies herbáceas exóticas. En estos bancos se observó además la presencia de C. tala durante las primeras cinco semanas del ensayo pero con una mortalidad del $100 \%$. Esto podría deberse a las condiciones edáficas menos apropiadas para el establecimiento de esta especie que presentan los cordones más alejados de la costa (Ribichich 1996). Estos resultados coinciden con las predicciones propuestas y podrían responder al efecto de fusión invasional descrito por Gioria et al. (2015) y Ferreras et al. (2015), a través del cual especies invasoras con dominancia estructural, como el ligustro, favorecen el establecimiento de otras especies exóticas.

Los bancos de semilla de los bosques con menor grado de invasión (primer cordón) se encontraron dominados por especies herbáceas, entre las que se encontraron especies exóticas y nativas. El banco de semillas del bosque conservado estuvo dominado por Stellaria media, herbácea exótica característica de agroecosistemas (Requesens y Madanes 1992). En el bosque semi-conservado, dominaron las semillas de especies nativas como O. setarius, Oxalis articulata Savigny e Hydrocotyle vulgaris L. Sin embargo, también se observó la presencia de especies exóticas como S. media y Parietaria debilis. Cabe señalar que en estos dos bosques, conservado y semi-conservado, no se observó la emergencia de plántulas de especies leñosas nativas presentes en la vegetación (S. buxifolia, J. rhombifolia, S. longifolius). Estos resultados coinciden con lo observado por Ferri et al. (2009) en bosques xéricos templados de la región, en los que los bancos de semillas estuvieron compuestos principalmente por especies herbáceas y sólo 3 especies leñosas y ninguna de ellas era una especie dominante en la vegetación. El mismo autor hace referencia a que entre las herbáceas, las especies exóticas aportaron relativamente más semillas que las especies nativas, coincidiendo con los resultados obtenidos en este trabajo.

Es importante destacar que en este trabajo solo se registró la presencia de tres especies arbóreas en los bancos de semillas (Celtis tala, Ligustrum lucidum y Acer negundo). Esto podría deberse a los distintos períodos de viabilidad que presenta cada semilla, ya que el banco de semillas analizado se corresponde al período de fructificación del año anterior de las principales especies nativas. A su vez, Thompson (1997) observó que los bancos de semillas de bosques maduros se componen principalmente de especies pioneras. Celtis tala presenta características de especie pionera, siendo reemplazada en la sucesión por Scutia buxifolia (Arturi y Goya 2004). Este atributo podría explicar la presencia de $C$. tala y la ausencia de $S$. buxifolia en los bancos de semillas, siendo ambas especies dominantes de los bosques estudiados.

La similitud total de especies entre el banco de semillas y la vegetación fue de $40 \%$, mayor a la reportada por otros autores en bosques templados. De acuerdo a Kalesnik et al. (2012), este valor se correspondería a un estado sucesional intermedio en bosques conservados. Sin embargo, este valor podría evidenciar un grado de invasión avanzado en bosques invadidos, con una alta presencia de las especies exóticas dominantes del dosel en el banco de semillas. En el análisis específico por bosque, los bosques conservado y ligustral presentaron los menores índices de similitud. En el caso del bosque conservado, este valor responde al estado sucesional tardío en el que se encuentra. En el ligustral esto puedo deberse a que, si bien el banco de semillas del ligustral estuvo dominado casi exclusivamente por L. lucidum, se registró también la presencia, y en algunos casos dominancia, de especies herbáceas nativas típicas del talar, como $O$. setarius. Cabe destacar que durante el período de germinación, las plántulas de L. lucidum fueron removidas a medida que germinaron, por lo que se facilitaría la germinación de otras especies que no necesariamente emergen en condiciones naturales. A su vez, se observó una gran diferencia en la diversidad entre los bosques analizados, alcanzando valores máximos en los sectores conservados del bosque y valores mínimos en sectores que presentan un alto grado de invasión (bosques ligustral e implantado). Además, en los bosques conservados se observó un mayor contenido de materia orgánica y menor $\mathrm{pH}$ que se asoció a una mayor riqueza y abundancia de especies herbáceas, y a una menor abundancia de L. lucidum. Esta asociación o relación podría responder a una alteración en la dinámica del proceso de descomposición y el ciclado de nutrientes de los bosques invadidos por el ligustro (Furey et al. 2014), que disminuirían el contenido de materia orgánica y alcalinizarían levemente el suelo llevándolo a valores neutros, hecho que ha sido observado previamente para la especie co-genérica $L$. sinense en bosques del sudeste de los Estados Unidos (Lobe et al. 2014). 
En este estudio, los bosques conservado y semi-conservado presentaron mayor diversidad y riqueza de especies y menores índices de invasión y cobertura de especies exóticas en todos los estratos, indicando que el proceso de invasión no ha alcanzado aún un grado avanzado. La baja diversidad y riqueza registradas para el ligustral y el bosque implantado, junto con índices de invasión superiores al $75 \%$ y coberturas de especies exóticas cercanas al $100 \%$, indica que el proceso de invasión provocó la alteración de la estructura y funcionamiento de estos bosques dominados por L. lucidum, y podrían ser considerados como neoecositemas (Kalesnik et al. 2012).

\section{CONCLUSIONES}

Los resultados obtenidos concuerdan con el modelo de fusión invasional, donde las invasiones afectan no solo la composición y abundancia de especies en la comunidad vegetal sino también la de los bancos de semillas. Los resultados obtenidos en este trabajo sugieren que el grado de invasión que presentan algunos de los bosques costeros de Buenos Aires ha superado el umbral de irreversibilidad, consolidándose como neoecosistemas dominados por L. lucidum. La invasión por L. lucidum ha modificado tanto la estructura vertical de las comunidades del bosque nativo como la relación de semillas de especies nativas y exóticas del banco de semillas. Además, estos nuevos tipos de bosques podrían alterar procesos funcionales de los bosques nativos que reemplazan como la producción de biomasa y hojarasca, y las tasas de descomposición.

\section{REFERENCIAS}

Albrecht H, M Pilgram. 1997. The weed seed bank of soil in a landscape segment in southern Bavaria. Plant Ecology 131: 31-43.

Aragon R, Groom M. 2003. Invasion by Ligustrum lucidum (Oleaceae) in NW Argentina: early Savage characteristics in different habitat types. Revista de Biología Tropical 51: 59-70.

Athor J. 2010. Parque Costero del Sur: Naturaleza, conservación y patrimonio cultural. Buenos Aires, Argentina. Fundación de Historia Natural "Felix Azara". 528 p.

Cabrera AL. 1976. Regiones fitogeográficas argentinas (Vol. 1). Enciclopedia argentina de agricultura y jardinería; Tomo 2 fasc. 1. Buenos Aires, Argentina. Acme. 85 p.

Cabrera AL. 1993. Manual de la flora de los alrededores de Buenos Aires. Buenos Aires, Argentina. Acme. 756 p.

Cagnoni M, AM Faggi, A Ribichich. 1996. La vegetación de la Reserva "El Destino" (Partido de Magdalena, Provincia de Buenos Aires). Parodiana 9(1-2): 25-44

Davies BE. 1974. Loss-on-ignition as an estimate of soil organic matter. Soil Science Society of America Journal 38(1): 150-151.

Escudero A, R Gavilán, A Rubio. 1994. Una breve revisión de técnicas multivariantes aplicables a Fitosociología. Botanica Complutensis 19: 9-38.

Ferreras AE, MA Giorgis, PA Tecco, MR Cabido, G Funes. 2015.
Impact of Ligustrum lucidum on the soil seed bank in invaded subtropical seasonally dry woodlands (Córdoba, Argentina). Biological Invasions 17: 3547-3561.

Ferri R, M Ceballos, N Vischi, E Heredia, A Oggero. 2009. Banco de semillas de un relicto de Espinal (Córdoba, Argentina). Iheringia 64: 93-100.

Furey C, PA Tecco, N Perez-Harguindeguy, MA Giorgis, M Grossi. 2014. The importance of native and exotic plant identity and dominance on decomposition patterns in mountain woodlands of central Argentina. Acta Oecologica 54: 13-20.

Gioria M, P Pyšek, L Moravcova. 2012. Soil seed banks in plant invasions: promoting species invasiveness and long-term impact on plant community dynamics. Preslia 84(2): 327350 .

Gioria M, V Jarošík, P Pyšek. 2014. Impact of invasions by alien plants on soil seed bank communities: emerging patterns. Perspective in Plant Ecology Evolution and Systematics 16:132-142.

Goya J, G Placci, MF Arturi, A Brown. 1992. Distribución y características estructurales de los Talares de la Reserva de Biosfera Parque Costero del Sur. Revista de la Facultad de Agronomía 68(1): 53-64.

Hoyos LE, GI Gavier-Pizarro, T Kuemmerle, EH Bucher, VC Radeloff, PA Tecco. 2010. Invasion of glossy privet (Ligustrum lucidum) and native forest loss in the Sierras Chicas of Córdoba, Argentina. Biological Invasions 12(9): 32613275 .

Kalesnik F, H Sirolli, M Collantes. 2012. Seed bank composition in a secondary forest in the Lower Delta of the Paraná River (Argentina). Acta Botánica Brasilica 27(1): 40-49.

Lobe JW, MA Callaham, PF Hendrixa, JL Hanula. 2014. Removal of an invasive shrub (Chinese privet: Ligustrum sinense Lour) reduces exotic earthworm abundance and promotes recovery of native North American earthworms. Applied Soil Ecology 83: 133-139.

Magurran AE. 1988. Ecological diversity and its measurements. Londres, Inglaterra. Chapman y Hall Press. 179 p.

Mueller-Dombois D, H Ellenberg. 1974. Aims and methods of vegetation ecology. New York, USA. John Willey and Sons. 547 p.

Requesens E y N Madanes. 1992. Organización de comunidades estacionales de malezas en el sudeste de la Provincia de Buenos Aires. Ecología Austral 2: 101-108.

Ribichich AM. 1996. Celtis tala Planchon (Ulmaceaes) seedling establishment on contrasting soils and microdisturbances: A greenhouse trial concerning adults' filed distribution pattern. Flora 191: 321-327.

Rodriguez N M y N Madanes. 2010. Efecto de la pava de monte en la dispersión y establecimiento de Ligustrum sinense (Lour.). Simposio científico académico delta del Paraná. Historia, Presente y futuro en los 10 años de la Reserva de la Biosfera del Delta del Paraná. San Fernando, Buenos Aires, Argentina.

Simpson RL, MA Leck, VT Parker. 1989. Seed banks: general concepts and methodological issues. In Leck MA, RL Simpson, VT Parker eds. Ecology of Soil Seed Banks. San Diego, Ca, USA. Academic Press. p. 3-8.

Thompson K. 1997. The soil seed banks of North West Europe: methodology, density and longevity (Vol. 1). Londres, Inglaterra. Cambridge University Press. 286 p. 
Vilá M. 2010. La investigación sobre invasiones por especies vegetales en la actualidad. In Fondo Europeo Agrícola de Desarrollo Rural ed. Especies exóticas invasoras en Andalucía. Talleres Provinciales 2004-2006. p. 73-76.
Zuloaga FO, O Morrone, M Belgrano. 2015. Catálogo de las Plantas Vasculares del Cono Sur (Argentina, Sur de Brasil, Chile, Paraguay y Uruguay). Consultado dic. 2015. Disponible en http://www.darwin.edu.ar/proyectos/floraargentina/fa.htm

Recibido: 27.04 .16

Aceptado: 03.08.16 